\title{
Synthesis of Nanoamorphous Germanium and Its Transformation to Nanocrystalline Germanium
}

\author{
Ömer Dag, ${ }^{*}$ Eric J. Henderson, and Geoffrey A. Ozin *
}

\begin{abstract}
A simple reaction between a mild reducing agent such as a trialkoxysilane and $G e^{I V}$ species such as germanium tetraalkoxides in a room-temperature water/ alcohol solution produces silica-coated ultrasmall (2-3 $\mathrm{nm}$ ) amorphous germanium nanoparticles (na-Ge/SiO$\left.{ }_{2}\right)$. The initial reaction involves the straightforward hydrolysis and condensation of the precursors, $\mathrm{Ge}\left(\mathrm{OCH}_{2} \mathrm{CH}_{3}\right)_{4}$ and $\left(\mathrm{CH}_{3} \mathrm{CH}_{2} \mathrm{O}\right)_{3} \mathrm{SiH}$, where the reaction rate depends on the water concentration in the reaction medium. These processes can be further accelerated by adding acid to the reaction medium or carrying out the reaction at higher temperatures. At low water contents (up to 50\% water/ethanol) and low acid concentrations, the reaction proceeds as a clear solution, and no precipitation is observed. The initially colorless clear solution progressively changes to pale yellow, yellow, orange, red, and finally dark red as the na-Ge particles grow. Evaporation of the solvent yields a reddish-brown powder/monolith consisting of na-Ge, embedded in an encapsulating amorphous silica matrix, na-Ge/SiO ${ }_{2}$. The formation of na-Ge proceeds extremely slowly and follows a first-order dependence on both water concentration and diameter of the na-Ge particles under the reaction conditions used. Annealing of the na-Ge/SiO 2 powder under an inert atmosphere at $600{ }^{\circ} \mathrm{C}$ produces ultrasmall germanium nanocrystals (nc-Ge) embedded in amorphous silica (nc-Ge/SiO 2 ). Freestanding, colloidally stable nc-Ge is obtained by chemical etching of the encapsulating silica matrix.
\end{abstract}

\section{Introduction}

The synthesis and study of size-, shape-, and surfacecontrolled semiconductor nanomaterials has provided a fountain of knowledge from which many nanotechnology

\section{Prof. Ö. Dag}

Bilkent University

Department of Chemistry

06800 Ankara, Turkey

E-mail: dag@fen.bilkent.edu.tr

Dr. E. J. Henderson, Prof. G. A. Ozin

Materials Chemistry and Nanochemistry Research Group

Center for Inorganic and Polymeric Nanomaterials

Chemistry Department

80 St. George Street

University of Toronto

Toronto, ON, M5S 3H6, Canada

E-mail: gozin@chem.utoronto.ca

DOI: 10.1002/smll.201101993 applications have sprung. ${ }^{1-4]}$ The main body of knowledge in this area currently embodies heavy-metal II-VI, IV-VI, and III-V semiconductors, exemplified by CdTe, PbS, and InAs nanomaterials, which have been shown to pose a health and safety hazard to researchers, manufacturers, and consumers. ${ }^{[5]}$ Because of these safety concerns it behoves the nanochemistry community to focus its research effort on the synthesis of semiconductor nanomaterials that are purportedly "green", such as group IV elemental semiconductors ${ }^{[6]} \mathrm{Si}$ and Ge, in particular, to explore the possibility that they might be able to replace toxic semiconductor nanomaterials in consumer products like solar cells, detectors, light-emitting diodes, lasers, sensors, and displays. ${ }^{[7]}$ Relative to heavy-metal semiconductor nanomaterials our knowledge of the synthesis of group IV semiconductors, especially Ge, is still in its infancy, even though it has been identified as an attractive nanomaterial for solar energy, near-infrared light emission, thermoelectric, and biomedical applications. Herein, we describe a new synthetic strategy for controlling and following the 
nucleation and growth of Ge from the nanoscale amorphous phase through to the crystalline form with and without a protective sheath of silica.

There are numerous reports on methods to prepare germanium nanocrystals (nc-Ge); many of these methods involve top-down fabrication strategies that require specialized reagents and equipment or high-temperature treatment to provide adequate crystallinity. ${ }^{[8,9]}$ Generally, these physical methods produce particles with substantial size distributions, undesirable for applications that exploit size-dependent electronic properties. Bottom-up solution-phase synthetic chemistry methods offer better control of the size and shape of the nanoparticles, but often do not provide the good crystallinity required for many applications, and require the use of longchain ligands and surfactants to stabilize the particle surface and control growth. ${ }^{[10-26]}$ Due to their ease of preparation, these solution-phase methods are also more accessible to many researchers and more amenable to scaling. As a result, their development is in great demand to prepare high-quality materials for detailed chemistry and physical studies, essential for their eventual integration into practical devices. Moreover, controlling the synthesis conditions in order to probe nucleation and growth of amorphous germanium (na-Ge) and its crystallization to nc-Ge is critical to obtain a detailed understanding of their reaction fundamentals and mode of formation, which will undoubtedly lead to better control of nanoparticle characteristics.

Room-temperature and elevated-temperature solution syntheses of nc-Ge using strong reducing agents have been well documented over the past several years. ${ }^{[8-24]}$ The germanium sources in these investigations are usually the germanium halides, $\mathrm{GeX}_{n}$, or organogermanes ${ }^{[23,24]}$ in which the $\mathrm{Ge}$ center is either in the $4^{+}$or $2^{+}$oxidation state (e.g., $\mathrm{GeCl}_{4}, \mathrm{GeBr}_{4}, \mathrm{GeI}_{2}$ ), and long-chain phosphines and alkenes are often used as surface protection ligands for nanoparticle stabilization. The reducing agents used in these investigations are usually the strong ones, such as $\mathrm{LiAlH}_{4}, \mathrm{NaBH}_{4}$, sodium, sodium naphthalide, butyllithium, and Ge Zintl salts. ${ }^{[2-24]}$ In these reactions, the strength of the reducing agent and the reduction reaction rate are related to each other, and usually these strong reducing agents react very quickly such that the reaction kinetics and nanocrystal growth rates cannot be explored and are not under fine control. Slowing these reduction steps and collecting kinetic information will be very beneficial for the synthesis of Ge nanoparticles and will gainfully contribute to the nanochemistry of these systems.

Herein, we demonstrate the first use of a mild reducing agent that can reduce $\mathrm{Ge}^{\mathrm{IV}}$ at room temperature and a controlled rate. The rate of reduction of the Ge precursor and the nucleation and growth of $\mathrm{Ge}$ nanoparticles can be effectively slowed down to almost a standstill and the kinetics of the reaction can be monitored using common laboratory spectroscopic techniques. Trialkoxysilanes, ( $\mathrm{RO})_{3} \mathrm{SiH}$ with a $\mathrm{Si}-\mathrm{H}$ site, are mild reducing agents toward germanium alkoxides $\left(\mathrm{Ge}(\mathrm{OR})_{4}\right)$. In this investigation $\left(\mathrm{CH}_{3} \mathrm{CH}_{2} \mathrm{O}\right)_{3} \mathrm{SiH}$ and $\mathrm{Ge}\left(\mathrm{OC}_{2} \mathrm{H}_{5}\right)_{4}$ are used as reducing agent and germanium source, respectively, to obtain 2-3 nm nanoparticles of na-Ge in a water/alcohol mixture. Upon solvent evaporation, na-Ge nanoparticles are embedded in an encapsulating $\mathrm{SiO}_{2}$ matrix, na-Ge/SiO 2 . This then allows subsequent thermally induced crystallization of the na-Ge to nc-Ge, which can then be liberated by chemical etching. This synthetic approach offers the benefits of both solution reduction and thermal solid-state methods.

\section{Results and Discussion}

We present details of the formation of Ge nanoparticles from a room-temperature solution reduction of $\mathrm{Ge}\left(\mathrm{OCH}_{2} \mathrm{CH}_{3}\right)_{4}$ with $\left(\mathrm{CH}_{3} \mathrm{CH}_{2} \mathrm{O}\right)_{3} \mathrm{SiH}$ and control and observe the transition from na-Ge to nc-Ge. Silicon hydrides have previously been investigated as hydride sources in organic reactions, and have also been used to reduce metal cations on silicon nanoparticle surfaces. ${ }^{[27-30]}$ Compared to the strong reducing agents typically used for solution synthesis of $\mathrm{Ge}$ nanoparticles, $\left(\mathrm{CH}_{3} \mathrm{CH}_{2} \mathrm{O}\right)_{3} \mathrm{SiH}$ is very mild and allows the reaction to proceed at a very slow and controlled rate. In ethanol or any other solvent without water, the $\left(\mathrm{CH}_{3} \mathrm{CH}_{2} \mathrm{O}\right)_{3} \mathrm{SiH}$ and $\mathrm{Ge}\left(\mathrm{OCH}_{2} \mathrm{CH}_{3}\right)_{4}$ precursors do not react. However, addition of $\left(\mathrm{CH}_{3} \mathrm{CH}_{2} \mathrm{O}\right)_{3} \mathrm{SiH}$ and $\mathrm{Ge}\left(\mathrm{OCH}_{2} \mathrm{CH}_{3}\right)_{4}$ to a water/ ethanol solution initiates hydrolysis and condensation reactions, followed by hydride transfer reactions to form polymeric species (represented with an empirical formula of $\left[\mathrm{GeSi}_{2}(\mathrm{H})_{2}(\mathrm{O})_{x}(\mathrm{OR})_{10-x}\right]_{n}$ (where Ge:2Si:2H reflects the initial composition of the synthesis mixture and $\mathrm{R}=\mathrm{H}$ or $\mathrm{C}_{2} \mathrm{H}_{5}$ ); see Scheme 1). The solution is initially clear and colorless, but gradually turns light yellow. This transition time can take a few minutes to a day depending on the temperature and water concentration of the mixture. If the mixture is left to age under ambient conditions, then within a few days to weeks the color gradually changes from yellow to orange, and eventually to dark red over time as the reaction progresses (see Figure 1).

The initially formed hydrolysis and condensation product $\left[\mathrm{GeSi}_{2}(\mathrm{H})_{2}(\mathrm{O})_{x}(\mathrm{OR})_{10-x}\right]_{n}$ slowly transforms into

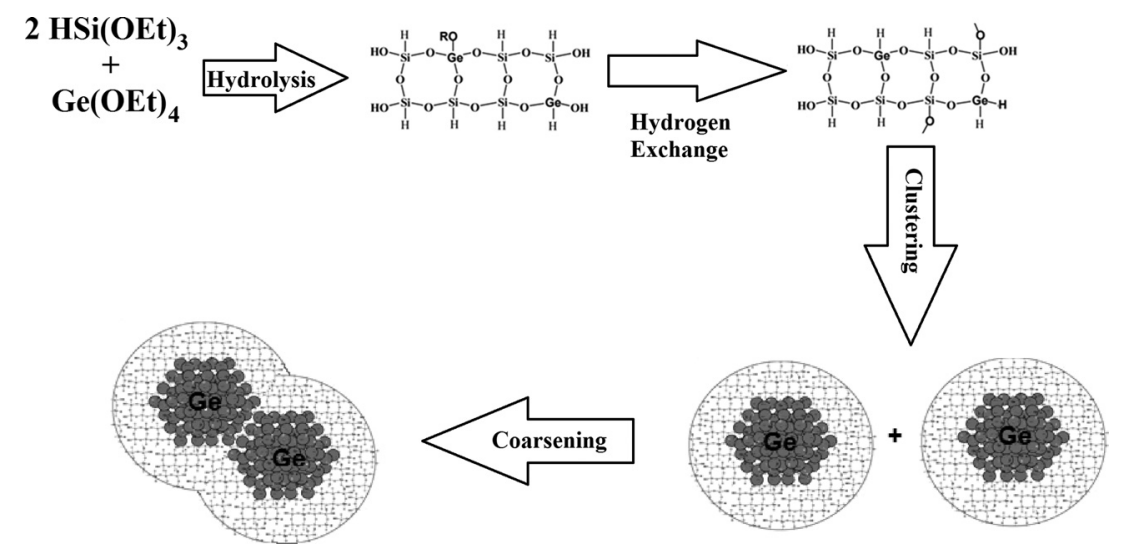

Scheme 1. Proposed hydrolysis, condensation, hydride exchange, and clustering processes involved in the nucleation and growth of na-Ge. 


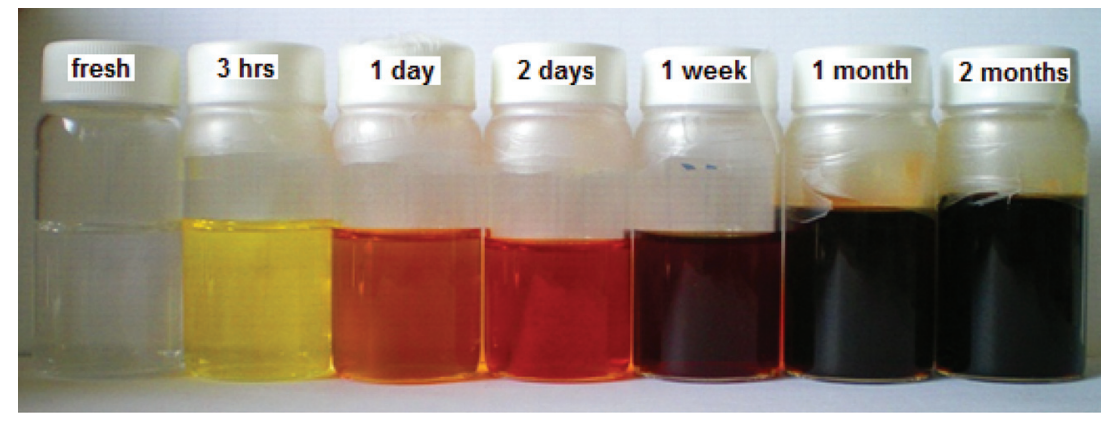

Figure 1. Time-dependent color changes in the synthesis solution.

na-Ge nanoparticles embedded in an amorphous silica matrix (Scheme 1). The color changes observed in the solution are due to the initial formation of Ge nuclei, and to the slow growth of the Ge nanoparticles. These particles are stable for months if the reaction vessel is tightly sealed from any air contact. The water content of the mixture can be varied to examine the water-dependent reaction kinetics (see (a)

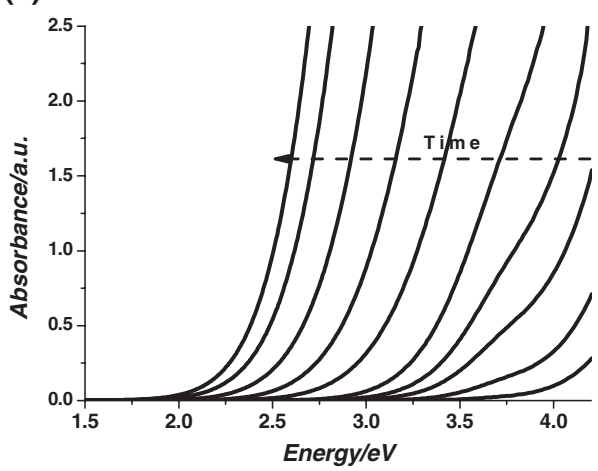

(b)

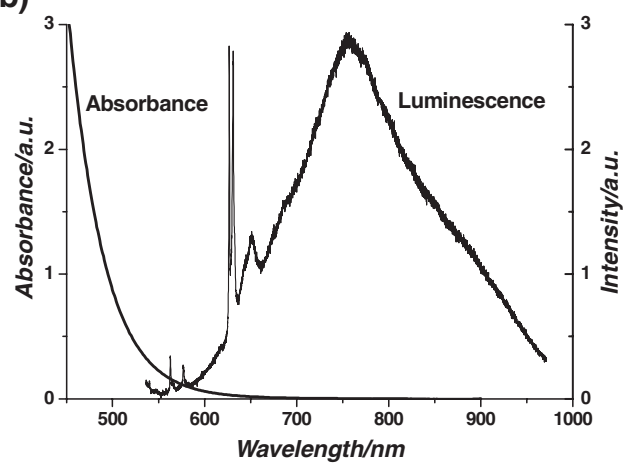

(c)

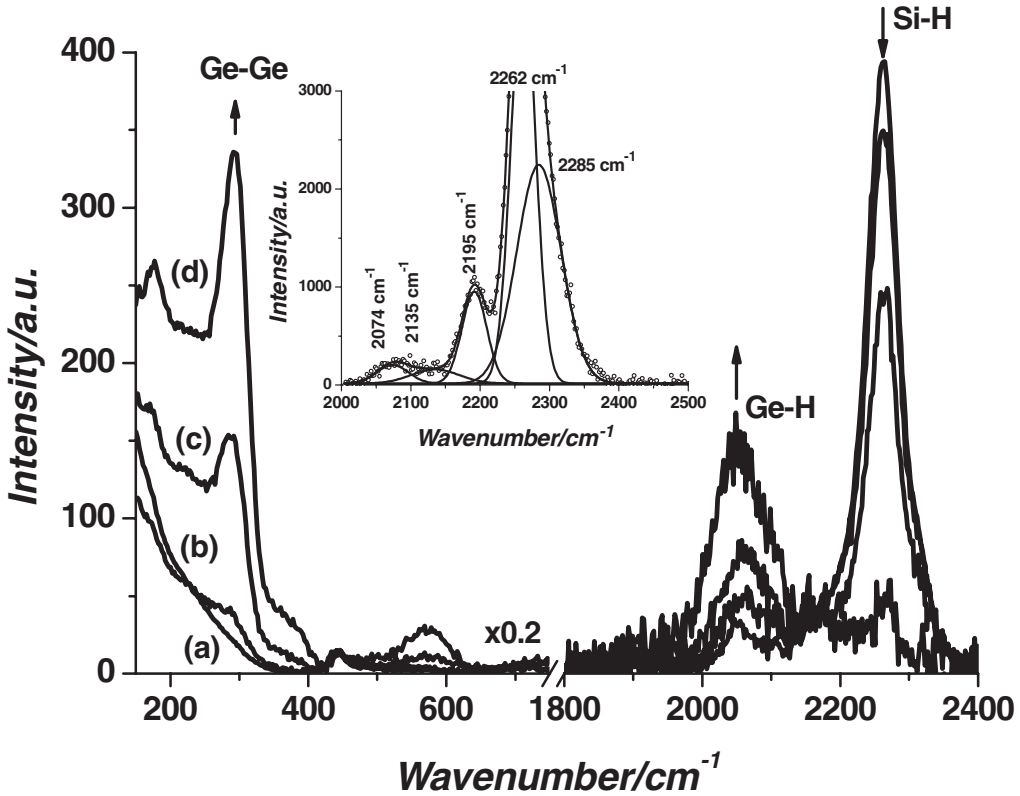

Figure 2. a) UV-vis-NIR absorption spectral changes with time. b) Absorption/PL spectra (excited at $532 \mathrm{~nm}$ ). c) Raman spectral changes with time a) $15 \mathrm{~min}$, b) $5 \mathrm{~h}, \mathrm{c}) 3$ days, and d) 3 weeks. Inset: the $v \mathrm{SiH}$ and $v \mathrm{GeH}$ stretching regions of a drop-cast sample at an intermediate stage of synthesis. below), but at concentrations over $50 \%$ $\mathrm{v} / \mathrm{v}$ water/ethanol the fast hydrolysis of $\mathrm{Ge}\left(\mathrm{OCH}_{2} \mathrm{CH}_{3}\right)_{4}$ leads to the precipitation of white $\mathrm{HSi}(\mathrm{O})_{1.5-x}(\mathrm{OR})_{2 x} \mathrm{GeO}_{2-x}(\mathrm{OR})_{2 x}$ species $\left(\mathrm{R}=\mathrm{C}_{2} \mathrm{H}_{5}\right.$ or $\left.\mathrm{H}\right)$. Interestingly, these white solid species are still reactive and over time the precipitate also changes color into a brownish yellow as Ge nanoparticles continue to form. The hydrolysis step is crucial, such that in the absence of water no reaction takes place, and the mixture of $\left(\mathrm{CH}_{3} \mathrm{CH}_{2} \mathrm{O}\right)_{3} \mathrm{SiH}$ and $\mathrm{Ge}\left(\mathrm{OCH}_{2} \mathrm{CH}_{3}\right)_{4}$ is stable for months. To support this proposal, we also examined two other silane precursors that cannot hydrolyze in our reaction conditions, namely $\left(\mathrm{CH}_{3}\right)_{3} \mathrm{SiH}$ and $\left(\left(\mathrm{CH}_{3} \mathrm{CH}_{2} \mathrm{O}\right)_{3} \mathrm{Si}\right)_{3} \mathrm{SiH}$ (notice no hydrolyzable site in the first and bulkiness in the second). These two precursors do not react with $\mathrm{Ge}\left(\mathrm{OCH}_{2} \mathrm{CH}_{3}\right)_{4}$ under any conditions examined in the present experiments, and no coloring due to formation of na-Ge was observed. Therefore, the initial hydrolysis and condensation that brings a $\mathrm{Si}-\mathrm{H}$ site in close proximity to $\mathrm{Ge}^{\mathrm{IV}}$ site(s) for subsequent transfer is crucial.

The formation and growth of na-Ge was followed using UV-vis absorption spectroscopy on samples with different $\left(\mathrm{CH}_{3} \mathrm{CH}_{2} \mathrm{O}\right)_{3} \mathrm{SiH} /$ $\mathrm{Ge}\left(\mathrm{OCH}_{2} \mathrm{CH}_{3}\right)_{4}$ molar ratios and water concentrations. Figure 2a shows the UV-visnear-infrared (NIR) absorption spectral changes during the na-Ge growth. A few minutes after the addition of the precursors, a shoulder appears in the spectra at around $325 \mathrm{~nm}$ that gradually shifts and intensifies in time, indicative of the growth of the Ge nanoparticles and the resulting narrowing of the highest occupied molecular orbital (HOMO)-lowest unoccupied molecular orbital (LUMO) gap. We also recorded the spectra of a 2 month aged solution (growth is complete) by diluting the mother liquor $300,150,100,75,50$, and 16 times (see Figure S1a,b, Supporting Information (SI)) to eliminate the possibility that the gradual red-shift is not due to increasing na-Ge concentration in the solution with time. While the normalized 
(a)

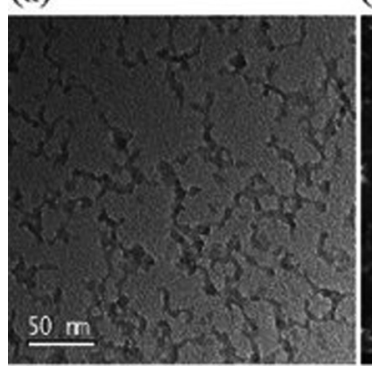

(b)

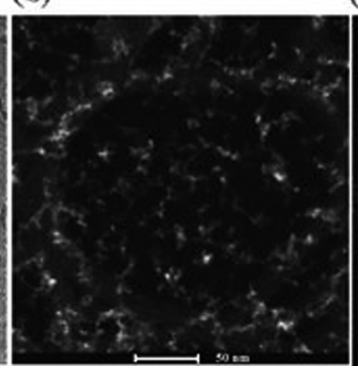

(c)

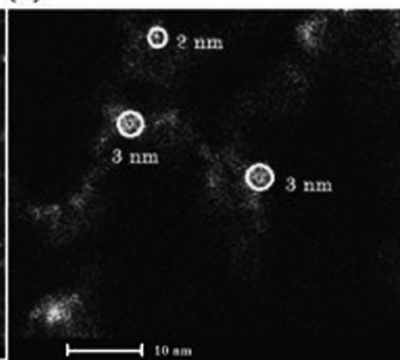

Figure 3. a) TEM and b,c) HAADF TEM images with different magnification of na-Ge thin films cast over a TEM grid.

spectra collected from these solutions completely overlap with each other, the spectra collected in time never overlap and display a gradual red-shift with increasing reaction time (compare SI, Figure S1a-d). Figure S1e shows two normalized spectra of a 2 month aged sample and a sample in an early stage of the reaction. Definitely, the spectrum of the aged sample displays a broad absorption that tails down to $1.7 \mathrm{eV}$, while the absorption vanishes at around $2.7 \mathrm{eV}$ in the fresh sample (compare spectra in SI, Figure S1e). Figure 2b and SI, Figure S2 display a Raman/photoluminescence (PL) spectrum, which is collected with a $532 \mathrm{~nm}$ excitation using the Raman setup in a methanol/water solution. The sharp lines, which are the Raman peaks of the solvent (methanol, $v \mathrm{CH}, \delta \mathrm{CH}$, etc.), and the broad feature at $756 \mathrm{~nm}$ with shoulders on each side (ca. 700 and $850 \mathrm{~nm}$ ) gradually increase in intensity, where the latter broadens and red-shifts with aging in the mother liquid most likely originates from the growing na-Ge particles (SI, Figure S2). The PL intensity and the solvent Raman signals have comparable intensities, thus indicating that the PL is extremely weak at this stage of the synthesis and not a concern of this investigation.

Further analysis of these samples at various stages of the reaction using Raman spectroscopy provides insight into the nucleation and growth process (Figure 2c). The peak at $2262 \mathrm{~cm}^{-1}$ and shoulder at $2286 \mathrm{~cm}^{-1}$ are due to the $\mathrm{Si}-\mathrm{H}$ vibrational modes in the reducing agent $\left(\left(\mathrm{CH}_{3} \mathrm{CH}_{2} \mathrm{O}\right)_{3} \mathrm{SiH}\right)$, and these decrease in intensity as the reaction progresses (Figure 2c). During the reaction, relatively weak peaks emerge at 2195, 2134, and 2074, and $2040 \mathrm{~cm}^{-1}$, attributed to $\mathrm{HGeO}_{3}, \mathrm{H}_{2} \mathrm{GeO}_{2}$, and $\mathrm{HGe}(\mathrm{Ge})_{x} \mathrm{O}_{3-x}$ species, ${ }^{[31]}$ respectively (Figure $2 \mathrm{c}$ ). At the same time, a new peak at around $287 \mathrm{~cm}^{-1}$, attributed to na-Ge, evolves and intensifies with the expansion of $\mathrm{Si}-\mathrm{H}$ and $\mathrm{Ge}-\mathrm{H}$ peaks. These observations suggest that the hydrolysis and condensation product, $\left[\mathrm{GeSi}_{2}(\mathrm{H})_{2}(\mathrm{O})_{x}(\mathrm{OR})_{10-x}\right]_{n}$, undergoes a hydride exchange reaction between the silicon and germanium centers to initiate the reduction process of $\mathrm{Ge}^{\mathrm{IV}}$ to $\mathrm{Ge}^{0}$ and thereupon further growth of na-Ge species.

Evaporation of the solvent from the pre-aged solution yields yellow-brown powders. During solvent evaporation under vacuum, the water concentration gradually increases, which results in the further condensation of the silicon alkoxide and silicon hydroxide species and the encapsulation of the na-Ge within a silica matrix. It is also important to note that the evaporation of a relatively fresh solution in a vacuum oven at $70{ }^{\circ} \mathrm{C}$ also produces dark red monolith. It means that a higher

temperature and gradually increasing water content (due to evaporation in a vacuum oven) of the medium significantly speeds up the reaction and reduces the overall reaction period.

The powder samples are X-ray amorphous at this stage. The lack of crystallinity in these samples was also verified using high-resolution transmission electron microscopy (HRTEM) and selectedarea electron diffraction (SAED), and no crystalline domains were observed.

The TEM images were collected using the benefits of the high-angle annular dark field (HAADF) mode to enhance the contrast originating from the na-Ge particles encapsulated within the silica matrix. Notice also that the casting of a drop of the mother liquid gives a thin silica film with embedded na-Ge particles. The TEM images show ultrafine aggregates of na-Ge nanoparticles (Figure 3a). In the HAADF TEM images (Figure $3 b, c)$ na-Ge particles as small as $2-3 \mathrm{~nm}$ are visible, as marked in Figure 3c. The HF-treated samples, however, display larger particles, most likely due to aggregation of the smaller na-Ge particles in their HAADF TEM images (see SI, Figure S3). No crystalline domains or crystallization under the electron beam were observed from these na-Ge particles. Note also that the na-Ge particles with or without silica coating are reactive to air and degrade in a few months upon exposure to ambient conditions (see the UV-vis-NIR spectra of the powder with time in SI, Figure S4).

The na-Ge nucleation and growth was followed using UVvis-NIR spectroscopy, as shown in Figure 2a, Figure 4, and Figure 5a in water/ethanol, water/methanol, and water/acetonitrile solutions. The absorption onset energies on the lowenergy side of each spectrum (obtained by extrapolating the absorption edge to zero absorbance on the low-energy side of each spectrum, see SI, Figure S1f) were plotted with respect to time at two different water concentrations (Figure 5b). The

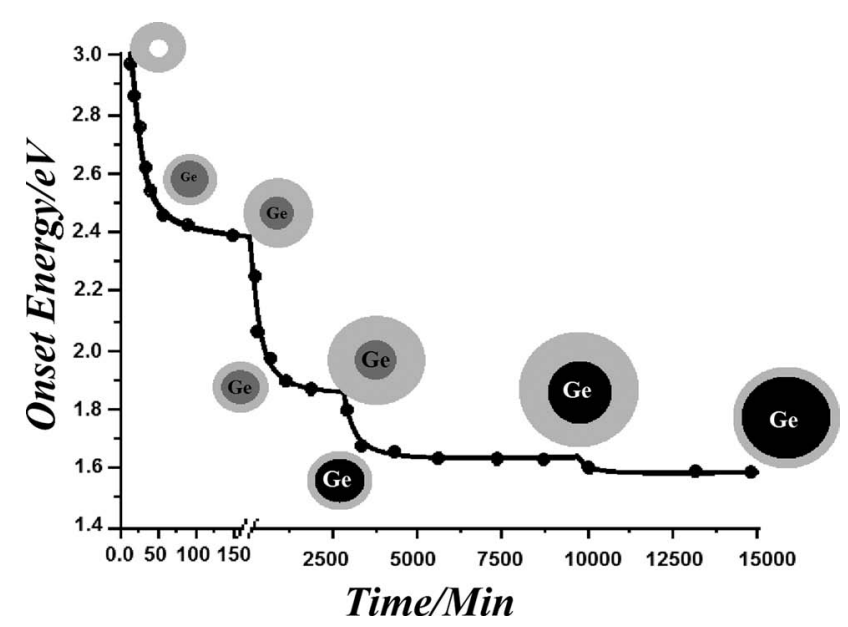

Figure 4. Nucleation and growth of na-Ge: the steps observed on the onset energies in time. 
(a)

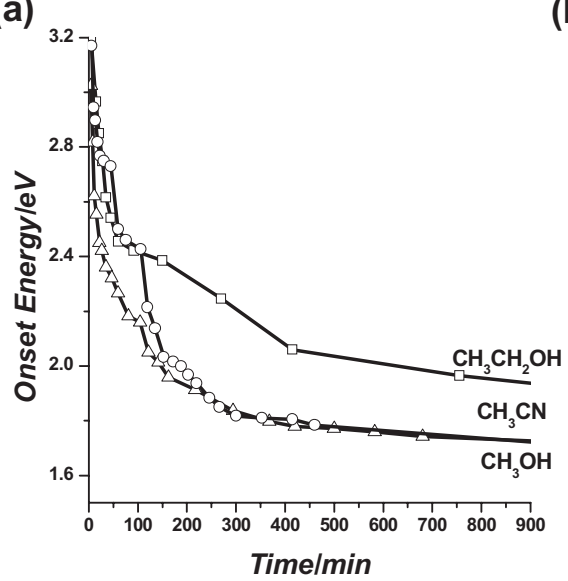

(b)

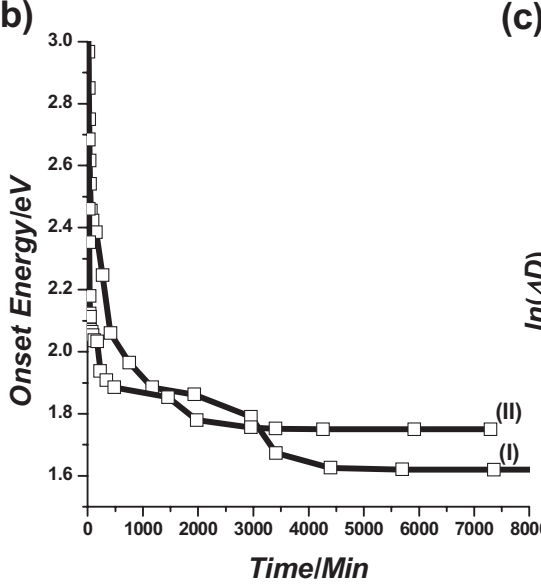

(c)

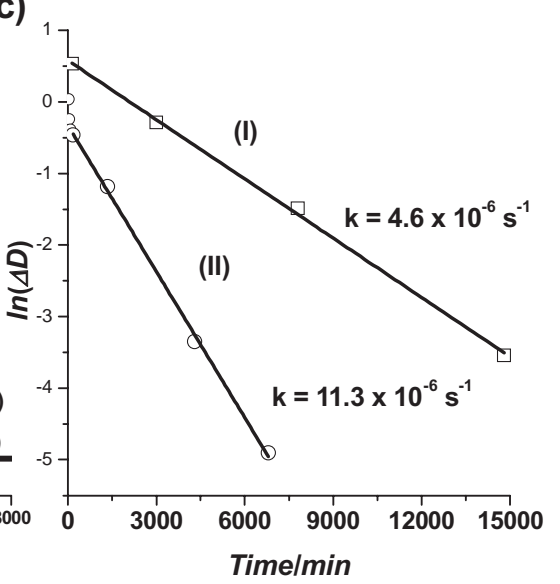

Figure 5. Nucleation and growth of na-Ge. a) Onset energy versus time plots in ethanol, acetonitrile, and methanol solutions. b) Onset energy versus time plots at two different water concentrations: I) 11.1 and II) $25.9 \mathrm{M}$. c) In( $\Delta D$ ) versus time plots at two different water concentrations: I) 11.1 and II) $25.9 \mathrm{M}$.

absorption edge gradually shifts to lower energy over time, thereby indicating the growth of the na-Ge. The onset energy undergoes an exponential decrease and subsequently reaches a plateau with increasing time, and then decreases again and reaches another plateau in all three solvents (Figure 5a). Figure 4 shows the steps observed from the absorption onset energies together with a possible primitive growth mechanism. In this proposal, there are two competing steps: hydrolysiscondensation of triethoxysilane and germanium tetraethoxide precursors to $\left(\mathrm{GeSi}_{2}(\mathrm{H})_{2}(\mathrm{O})_{x}(\mathrm{OR})_{10-x}\right)_{n}$ species and the subsequent reduction-clustering process. The clustering (or formation of na-Ge) step is most likely taking place at the core of these particles, driven by the hydrolysis, condensation, and hydride-exchange steps occurring in the shell of the particles. These steps are schematically shown in Scheme 1. Note also that there is no hydrogen evolution or $\mathrm{pH}$ rise during the reduction reaction. The reaction carried out in excess reducing agent produced $\left(\mathrm{O}_{1.5} \mathrm{SiH}\right)_{n}$ species in the samples, as evidenced from the ${ }^{29} \mathrm{Si}$ high-power decoupling (HPDEC) magic angle spinning (MAS) NMR spectrum. SI, Figure S5 shows the ${ }^{29} \mathrm{Si}$ HPDEC MAS NMR spectrum of a sample prepared using a 4:1 mole ratio of $\left(\mathrm{CH}_{3} \mathrm{CH}_{2} \mathrm{O}\right)_{3} \mathrm{SiH}$ and $\mathrm{Ge}\left(\mathrm{OCH}_{2} \mathrm{CH}_{3}\right)_{4}$ after complete reduction. The $29 \mathrm{Si} \mathrm{NMR}$ spectrum displays peaks at $-76.7,-84.7$, and $-94.5,-101.5$, and $-110.1 \mathrm{ppm}$ originating from $\mathrm{T} 2\left(\mathrm{HSi}(\mathrm{O})_{2}(\mathrm{OH})\right)$ and $\mathrm{T} 3$ $\left(\mathrm{HSiO}_{3}\right)$ (total $\left.52.5 \%\right)$ and $\mathrm{Q} 2\left(\mathrm{O}_{2} \mathrm{Si}(\mathrm{OH})_{2}\right), \mathrm{Q} 3\left(\mathrm{O}_{3} \mathrm{SiOH}\right)$, and Q4 $\left(\mathrm{O}_{4} \mathrm{Si}\right)$ (total $\left.47.5 \%\right)$ sites, respectively, due to polymerized unreacted and reacted reducing agent. These ratios ensure $95 \%$ of $\mathrm{Ge}^{\mathrm{IV}}$ is reduced to $\mathrm{Ge}^{0}$. The overall reaction can be written as follows:

$$
\begin{aligned}
& 2\left(\mathrm{CH}_{3} \mathrm{CH}_{2} \mathrm{O}\right)_{3} \mathrm{SiH}+\mathrm{Ge}\left(\mathrm{OCH}_{2} \mathrm{CH}_{3}\right)_{4}+4 \mathrm{H}_{2} \mathrm{O} \\
& \rightarrow \text { na-Ge- }\left(\mathrm{SiO}_{2}\right)_{2}+10 \mathrm{CH}_{3} \mathrm{CH}_{2} \mathrm{OH}
\end{aligned}
$$

All of the reactions in this investigation were carried out in the presence of $\left(\mathrm{CH}_{3} \mathrm{CH}_{2} \mathrm{O}\right)_{3} \mathrm{SiH}$ and $\mathrm{Ge}\left(\mathrm{OCH}_{2} \mathrm{CH}_{3}\right)_{4}$ (4:1) to ensure complete reaction and to further stabilize the $\mathrm{Ge} 0$ species in the solution. As previously mentioned, the initial color of the solution depends on the concentration of water in the reaction medium. Increasing the water concentration enhances the hydrolysis and condensation steps. Moreover, at high water concentrations it is likely that more seeds are created, which leads to smaller na-Ge particles as for the lower water concentrations (compare the plots in Figure 5b). Figure 5b shows two sets of data with 11.1 and 25.9 м water content. The water concentrations were kept high to speed up the process and to keep its concentration constant during the process to evaluate water-independent rate constants. The plots in Figure $5 \mathrm{~b}$ and $\mathrm{c}$ were obtained using an approximation for the nanoparticle size that was calculated using the onset energies at the plateau, where the shift on the absorption edge almost stops, and the HOMOLUMO energy and the nanoparticle size relation. ${ }^{[32]}$ The effective mass model (EMM) predicts that the bandgap of semiconductors scales with the particle diameter according to the following equation:

$E(d)=E_{\mathrm{g}}+2 \hbar^{2} \pi^{2} d^{2 *}\left(1 / m_{\mathrm{e}}+1 / m_{\mathrm{h}}\right)$

where $E_{\mathrm{g}}$ is the bulk bandgap, $d$ is the diameter of the nanoparticles, and $m_{\mathrm{e}}$ and $m_{\mathrm{h}}$ are the effective masses of electron and hole, respectively. The bandgap of nc-Ge can be predicted from $E(d)=0.66+16.8 / d^{2}$, where the bulk bandgap of nc-Ge is $0.66 \mathrm{eV}, 16.8$ is all the constants in the expression for nc-Ge, $d$ is the diameter of nc-Ge, and $m_{\mathrm{e}}$ and $m_{\mathrm{h}}$ are 0.123 and $0.33,{ }^{[31]}$ respectively; for the na-Ge, $E(d)=1.05+$ $10.3 / d^{2}$, where the bulk bandgap of na-Ge, $m_{\mathrm{e}}$, and $m_{\mathrm{h}}$ are $1.05 \mathrm{eV}, 0.22$, and $0.43,{ }^{[33,34]}$ respectively (see Figure S6). Note also that the nanoparticles selected for size analysis are the largest ones in the solution, which are formed first and therefore they have the absorption at the lowest energy.

The logarithm of particle size change $\Delta D$ (where $\Delta D$ is the difference in diameter of the na-Ge particles at time infinity and the end of each step) versus time gives a linear plot, characteristic of a first-order reaction (Figure 5c). The slope of the plot gives the water-dependent rate constant $k=$ $\mathrm{k}^{\prime}\left[\mathrm{H}_{2} \mathrm{O}\right]^{n}$, the $k$ values at two different water concentrations 

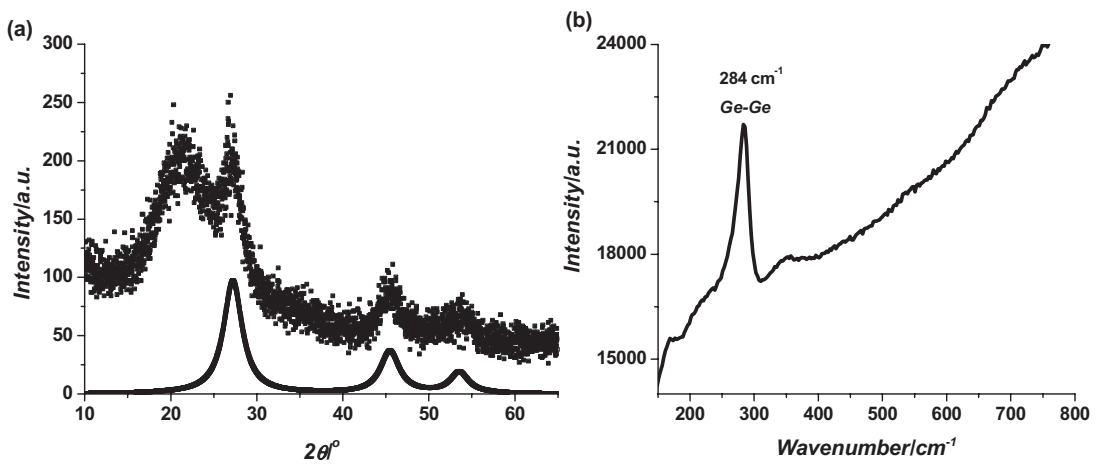

Figure 6. a) PXRD pattern and b) Raman spectrum of nc- $\mathrm{Ge} / \mathrm{SiO}_{2}$.

being given in the plots (see Figure $5 \mathrm{c}$ ). The ratio of $k$ values at these two water concentrations gives $n$, which is equal to 1.0; therefore the nanoparticle growth also has a first-order dependence on the water concentration in the media and the na-Ge diameter changes with a rate constant, $k^{\prime}$, of $4.26 \times$ $10^{-7} \mathrm{~s}^{-1}$ under our reaction conditions. Both plots, at two different water concentrations, give the same rate constant. Therefore the rate expression for the growth can be written as $k^{\prime}\left[\mathrm{H}_{2} \mathrm{O}\right](D)$.

The powder samples of silica-encapsulated na-Ge can then be annealed under $\mathrm{N}_{2}$ at $600-700{ }^{\circ} \mathrm{C}$ to crystallize the $\mathrm{Ge}$ particles. This is a distinct advantage of this synthetic method, in that it allows very fine tuning of the particle size via a slow solution route, and then allows high-temperature crystallization owing to the inert encapsulating matrix. The powder X-ray diffraction (PXRD) pattern of the nc-Ge/SiO 2 exhibits broad reflections at $2 \theta=27.2,45.5$, and $53.6^{\circ}$, characteristic of the (111), (220), and (311) planes of diamond structure Ge, respectively (Figure 6a). The approximate nanocrystal size can be calculated from the peak broadening using Scherrer's equation, and was estimated as approximately $4 \mathrm{~nm}$. The Raman spectrum of the $\mathrm{nc}-\mathrm{Ge} / \mathrm{SiO}_{2}$ displays a relatively
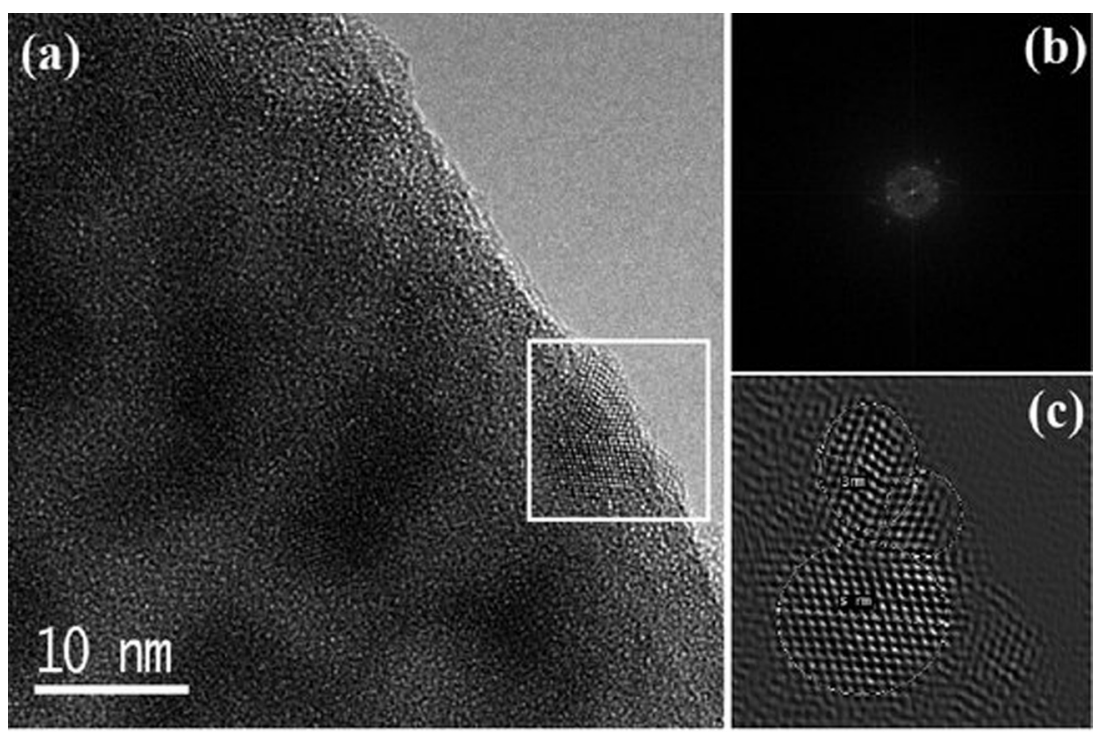

Figure 7. a) TEM image, b) FFT of the area selected in (a), and c) back FFT of selected area of nc- $-\mathrm{Ge} / \mathrm{SiO}_{2}$. sharp peak at $284 \mathrm{~cm}^{-1}$, characteristic of the transverse optical phonon in crystalline $\mathrm{Ge}$, which is quite red-shifted from the bulk value at around $300 \mathrm{~cm}^{-1}$ due to the phonon confinement effect ${ }^{[35]}$ (Figure 6b). Using a phonon confinement $\operatorname{model}^{[32]}$ and the Raman transverse optical (TO) mode, the size of the nc-Ge was calculated to be less than $3.0 \mathrm{~nm}$. However, the PXRD, UV-vis-NIR absorption spectroscopy, and TEM measurements show that the average particle size is around $4.0 \mathrm{~nm}$. The slight inconsistency in the particle size calculated using Raman measurements may lie in the phonon confinement model used. ${ }^{[35]}$

The TEM images collected from the nc-Ge/SiO 2 show that the nc-Ge nanoparticles are crystalline, and homogeneously distributed in the silica matrix with a size distribution between 3 and $5 \mathrm{~nm}$ (Figure 7a and SI, Figure S7). The energy-dispersive X-ray (EDX) data of the samples show O, Si, and Ge signals with $\mathrm{Si} / \mathrm{Ge}$ intensity ratio of 0.25 , comparable to the initial composition of the synthesis solution (SI, Figure S8). Figure $7 \mathrm{~b}$ shows the fast Fourier transform (FFT) of nc-Ge; the spots, equivalent to diffraction spots, originate from (111), (220), and (311) lattice planes of diamond structure Ge. The unit cell parameter calculated from these spots is consistent with the bulk unit cell constant of diamond-type Ge, approximately $5.658 \AA .{ }^{[36]}$ The image (Figure 7c) generated by back FFT of the region marked in Figure 7a clearly shows the lattice fringes and spacing originating from the (111) planes of the diamond structure nc-Ge nanoparticles. The nonspherical particles are most likely due to oriented growth of two or three particles during the annealing step, as seen in the images shown in Figure 7 and SI, Figure S7.

The nc-Ge/SiO 2 samples were carefully etched using $40 \%$ aqueous HF solution to selectively remove the encapsulating silica matrix. The liberated nc-Ge nanoparticles can be dispersed in ethanol and are stable for weeks. After this time the nanoparticles aggregate and precipitate as black fine powders. The surface chemistry of Ge etched in HF solutions is not well understood, and most likely involves the formation of hydrides, alkoxides, hydroxides, and oxide species. The UVvis-NIR absorption spectrum of the dark clear solution gives a broad absorption tailing down to $880 \mathrm{~nm}$ (see Figure 8a). The absorption edge was fitted using the indirect electronic bandgap relation to evaluate $E_{\mathrm{g}}$ to be $1.42 \mathrm{eV}^{[37]}$ (see inset in Figure 8a). The etched nc-Ge particles were further analyzed using X-ray photoelectron spectroscopy (XPS), XRD, and TEM. The XPS spectrum shows both $\mathrm{Ge}^{0}$ (nc-Ge) and $\mathrm{Ge}^{\mathrm{IV}}\left(\mathrm{GeO}_{2}\right)$, thus indicating oxidation of the nanoparticle surface (Figure 8b). This is consistent with our model of the surface chemistry for these 
(a)

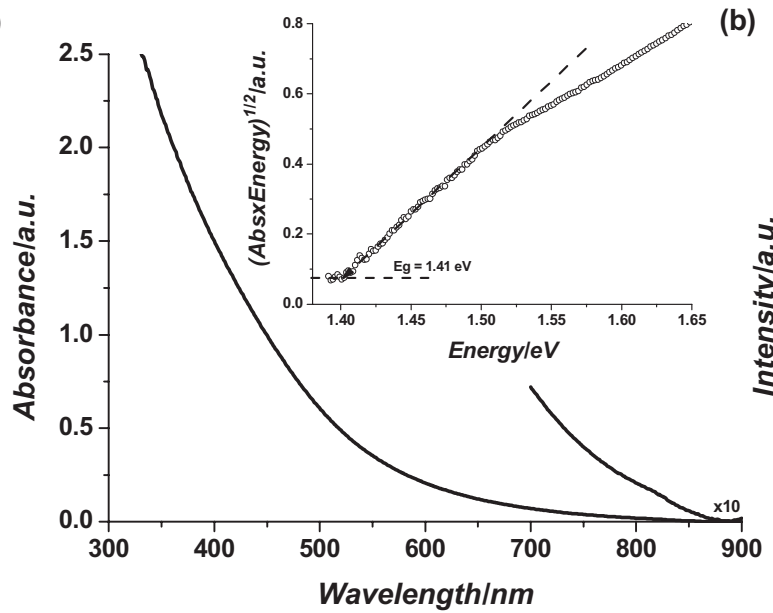

(b)

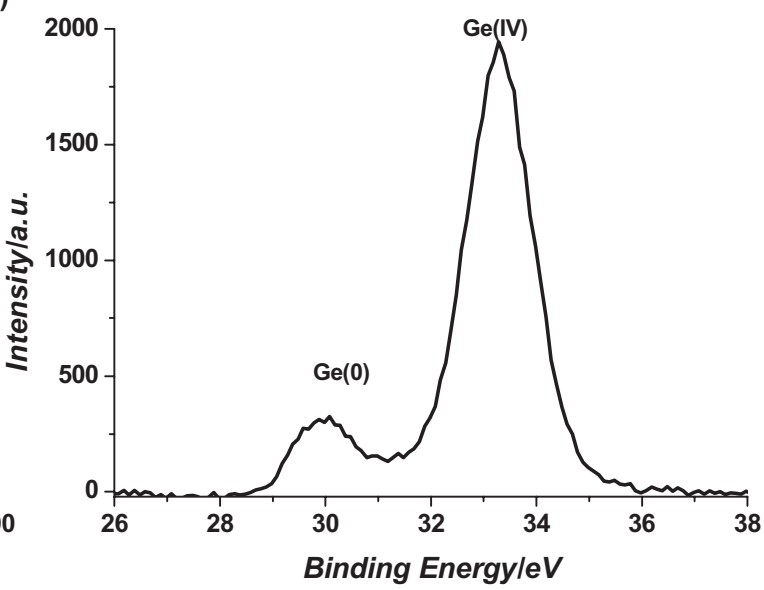

Figure 8. a) UV-vis-NIR absorption spectra (inset: plot for the indirect electronic bandgap fitting of the absorption edge) and b) XPS spectrum of nc-Ge.

nanocrystals. We also measured the ultraviolet photoelectron spectrum of the sample and determined the absorption onset that gives a take-off energy of $0.66 \mathrm{eV}$ above the Fermi level, which corresponds to the valance band edge (SI, Figure S9). The shift from the bulk value of $0.16 \mathrm{eV}^{[38]}$ is consistent with the bandgap evaluated from UV-vis-NIR data and quantum confinement effects.

The TEM images of the sample show crystalline Ge nanoparticles with an average particle size of about $3.0-5.0 \mathrm{~nm}$, which is consistent with the size obtained from the electronic band-gap value. The nc-Ge nanoparticles are better resolved, most likely due to removal of the silica over-layer (Figure 9 and SI, Figure S10). The lattice fringes and spacings are consistent with nc-Ge (see Figure 9). Note also that the etched samples show only a Ge signal in the EDX spectrum. This is also consistent with the XPS survey scan of the same sample, which shows no Si- or F-related peaks after $\mathrm{HF}$ etching (SI, Figure S11).
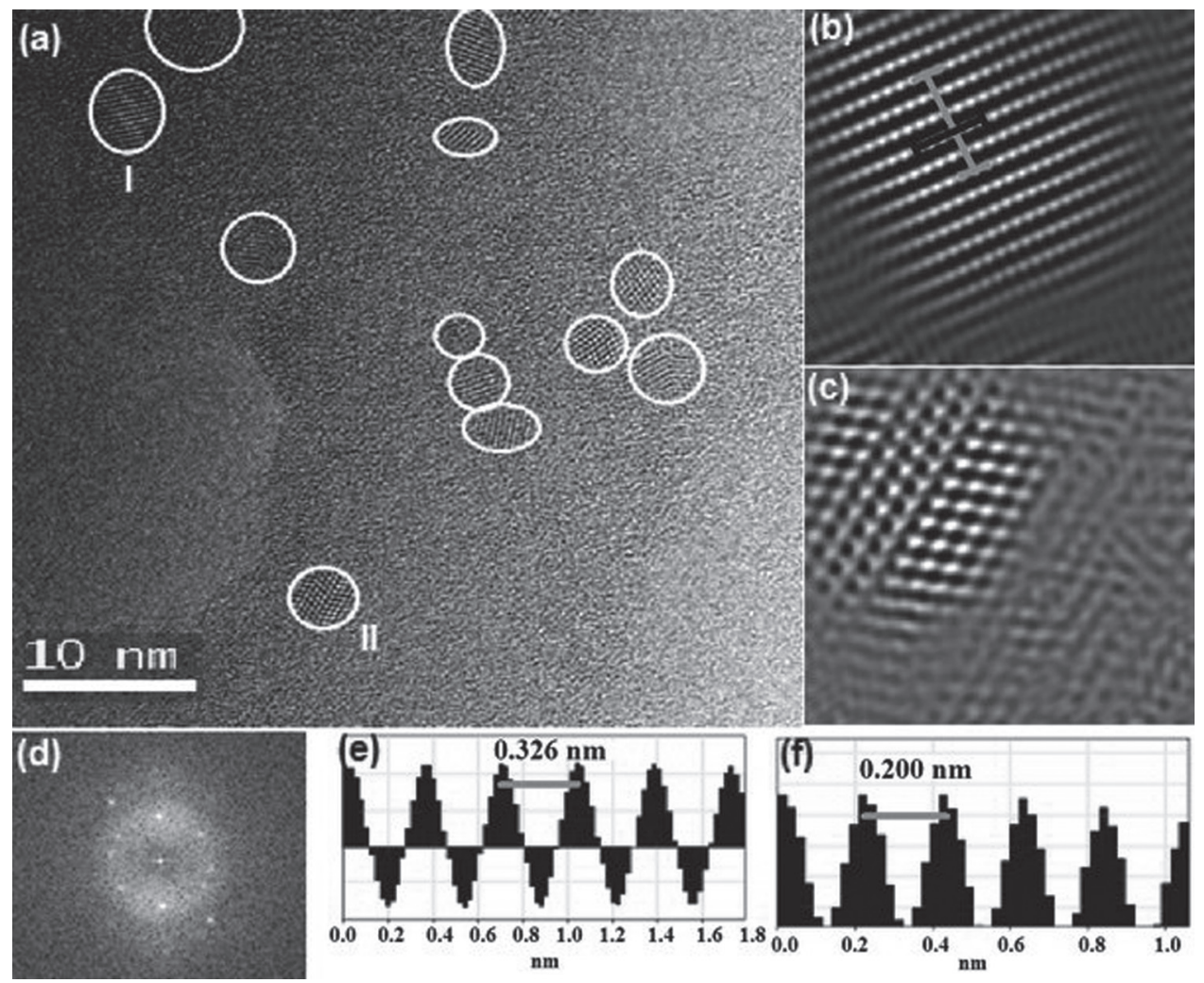

Figure 9. a) TEM image of nc-Ge after HF etching; b) the inverse FFT of region I in panel (a); c) inverse FFT of region II in panel (a); d) FFT of region I in panel (a); line scans of e) light and f) dark lines in panel (b). 


\section{Conclusion}

In summary, na-Ge/SiO ${ }_{2}$, nc-Ge/SiO ${ }_{2}$, and nc-Ge can be prepared using trialkoxysilane as a mild reducing agent and precursor. The $\mathrm{Ge}^{\mathrm{IV}}$ sol-gel product is reduced by $\mathrm{SiH}$ sites through a hydride exchange, and then transforms into na-Ge nanoparticles. This is also consistent with our previous investigation of the $\mathrm{Si}-\mathrm{H}$ units of periodic mesoporous hydrido silica as reducing sites for reduction of $\mathrm{Ag}^{+}$ion to produce Ag nanocrystals at room temperature. ${ }^{[39]}$ The likely driving force for this process is the formation of a shell of $\left[\mathrm{GeSi}_{2}(\mathrm{H})_{2}(\mathrm{O})_{x}(\mathrm{OR})_{10-x}\right]_{n}$ species that feeds the growing na-Ge core of the particles. The reaction proceeds with a first-order dependence on both water and the diameter of the na-Ge, with a rate constant of $4.26 \times 10^{-7} \mathrm{~s}^{-1}$. The na-Ge/SiO nanoparticles can be converted into silica-embedded crystalline germanium nanocrystals $\mathrm{nc}-\mathrm{Ge} / \mathrm{SiO}_{2}$ at around $600{ }^{\circ} \mathrm{C}$ under $\mathrm{N}_{2}$ atmosphere. The silica shell can subsequently be etched away to yield colloidally stable nc-Ge particles. The reaction of $\mathrm{Ge}\left(\mathrm{OCH}_{2} \mathrm{CH}_{3}\right)_{4}$ and $\left(\mathrm{CH}_{3} \mathrm{CH}_{2} \mathrm{O}\right)_{3} \mathrm{SiH}$ in a water/ alcohol mixture produces silica-capped $\mathrm{Ge}$ nanoparticles that remain as a clear solution for several months. Because of this long-term colloidal stability of na-Ge, these dispersions can be employed as a "Ge-Ink" and spin-coated onto various substrates to make silica-encapsulated na-Ge thin film, which on annealing under an inert atmosphere yields nc-Ge/SiO for thin-film applications.

\section{Experimental Section}

Preparation of na-Ge Nanoparticles: In a typical synthesis, $\mathrm{Ge}\left(\mathrm{OCH}_{2} \mathrm{CH}_{3}\right)_{4}(0.11 \mathrm{~mL})$ and $\left(\mathrm{CH}_{3} \mathrm{CH}_{2} \mathrm{O}\right)_{3} \mathrm{SiH}(0.36 \mathrm{~mL})$ were added to ethanol $(10 \mathrm{~mL})$ and water $(\times \mathrm{mL}, 0.1 \leq \times \leq 10 \mathrm{~mL})$ in a $25 \mathrm{~mL}$ vial which was then sealed with parafilm. The solution was kept at room temperature under ambient conditions. The clear and colorless solution turned yellow in a few minutes to a few days depending on the water concentration of the reaction mixture, and gradually darkened in color. After the color of the solution became dark red, the solvent was evaporated under vacuum using a Schlenk line. The yellow-brown na- $\mathrm{Ge} / \mathrm{SiO}_{2}$ powder was subsequently collected and kept in an airtight container until use. The powder and solution started degrading upon exposure to air.

Crystallization of na-Ge/SiO 2 to $n c-G e / \mathrm{SiO}_{2}$ Particles: The na- $\mathrm{Ge} / \mathrm{SiO}_{2}$ powder $(250 \mathrm{mg})$ was placed in a quartz reaction boat and heated to $600{ }^{\circ} \mathrm{C}$ under flowing $\mathrm{N}_{2}$ with a heating rate of $10^{\circ} \mathrm{C} \mathrm{min}-1$. The temperature was maintained at $600{ }^{\circ} \mathrm{C}$ for $1 \mathrm{~h}$ under flowing $\mathrm{N}_{2}$ and then cooled to room temperature. The dark brown nc- $\mathrm{Ge} / \mathrm{SiO}_{2}$ was placed into a sealed vial for later use.

Etching $n c-G e / \mathrm{SiO}_{2}$ : To liberate the nc-Ge from the encapsulating $\mathrm{SiO}_{2}$ matrix, the oxide was etched in a mixture of ethanol and aqueous HF. In a typical experiment, nc-Ge/SiO 2 (200 mg) was stirred in a mixture of water $(3 \mathrm{~mL})$, ethanol $(3 \mathrm{~mL})$, and $48 \%$ $\mathrm{HF}(\mathrm{aq})(3 \mathrm{~mL})$ for $15 \mathrm{~min}$. The freestanding nc-Ge particles were isolated by centrifugation and washed twice each with water and ethanol and dispersed in ethanol.

Etching na-Ge/SiO ${ }_{2}$ : The liberation of na-Ge was similar to that of $\mathrm{nc}-\mathrm{Ge}$, in which na-Ge/SiO $\mathrm{S}_{2}(100 \mathrm{mg})$ was etched in water $(3 \mathrm{~mL})$, ethanol $(3 \mathrm{~mL})$, and $48 \% \mathrm{HF}(\mathrm{aq})(3 \mathrm{~mL})$ for $5 \mathrm{~min}$.
Isolation and purification of freestanding na-Ge was as described above.

Material Characterization: Raman spectroscopy was performed using a $532 \mathrm{~nm}$ diode laser and calibrated using a crystalline silicon wafer. PXRD patterns of all samples were obtained on lowintensity-background substrates and acquired with a Siemens D5000 instrument using $\mathrm{Cu}_{\mathrm{K} \alpha}$ radiation $(\lambda=0.15418 \mathrm{~nm})$. HRTEM imaging and EDX spectroscopy were performed at the Canadian Centre for Electron Microscopy at McMaster University using an FEI Titan 80-300 keV electron microscope. TEM samples were prepared by drop-casting from ethanol solutions onto carbon-coated copper grids. XPS spectra were acquired using a Thermo Scientific Theta Probe utilizing monochromatic $\mathrm{Al}_{\mathrm{K} \alpha}$ radiation. UV-vis absorption spectra were collected in transmittance mode using the mother solutions of na-Ge and ethanol solutions of nc-Ge (etched samples) by using a Perkin-Elmer Lambda 900 spectrophotometer. The UV-vis-NIR absorption spectra of the solid samples were recorded in diffuse reflectance mode using the same equipment and a diffuse reflectance attachment.

\section{Supporting Information}

Supporting Information is available from the Wiley Online Library or from the author.

\section{Acknowledgements}

GAO is the Government of Canada Research Chair in Materials Chemistry and Nanochemistry. He thanks the Natural Sciences and Engineering Research Council (NSERC) of Canada for strong and sustained financial support of his research. ÖD thanks Bilkent University and the Turkish Academy of Science for financial support. We thank J. E. Lofgreen, Dr. S. Petrov, Dr. P. M. Brodersen, and Dr. C. Andrei for NMR, PXRD, XPS, UPS, and TEM measurements, respectively.

[1] G. A. Ozin, A. C. Arsenault, L. Cademartiti, Nanochemistry: A Chemical Approach to Nanomaterials, 2nd ed., Royal Society of Chemistry, Cambridge, 2009.

[2] L. Cademartiri, G. A. Ozin, Phil. Trans. R. Soc. A 2010, 368, 4229.

[3] C. B. Murray, D. J. Norris, M. G. Bawendi, J. Am. Chem. Soc. 1993, $115,8706$.

[4] G. M. Whiteside, Small 2005, 1, 172.

[5] N. Lewinski, V. Colvin, R. Drezek, Small 2008, 4, 26.

[6] J. Y. Fan, P. K. Chu, Small 2010, 6, 2080.

[7] a) Z. H. Lu, D. J. Lockwood, J. M. Baribeau, Nature 1995, 378, 258; b) S. N. Baker, G. A. Baker, Angew. Chem., Int. Ed. 2010, 49, 6726; c) D. P. Puzzo, E. J. Henderson, M. G. Helander, Z. B. Wang, G. A. Ozin, Z. H. Lu, Nano Lett. 2011, 11, 1585.

[8] Y. Maeda, N. Tsukamota, Y. Yazawa, Y. Kanemitsu, Y. Masunoto, Appl. Phys. Lett. 1991, 59, 3168.

[9] Y. Nakamura, K. Watanabe, Y. Fukuzawa, M. Ichikawa, Appl. Phys. Lett. 2005, 87, 133119.

[10] D. C. Lee, J. M. Pietryga, I. Robel, D. J. Werder, R D. Schaller, V. I. Klimov, J. Am. Chem. Soc. 2009, 131, 3436.

[11] D. A. Ruddy, J. C. Johnson, E. R. Smith, N. R. Neale, ACS Nano 2010, 4, 7459. 
[12] A. Kornowski, M. Giersig, R. Vogel, A. Chemseddine, H. Weller, Adv. Mater. 1993, 5, 634.

[13] J. R. Heath, J. J. Shiang, A. P. Alivisatos, J. Chem. Phys. 1994, 101, 1607.

[14] B. R. Taylor, S. M. Kauzlarich, Chem. Mater. 1999, 11, 2493.

[15] D. Gerion, N. Zaitseva, C. Saw, M. F. Casula, S. Fakra, T. Van Buuren, G. Galli, Nano Lett. 2004, 4, 597.

[16] T. Hanrath, B. A. Korgel, J. Am. Chem. Soc. 2004, 126, 15466.

[17] X. M. Lu, B. A. Korgel, K. P. Johnston, Chem. Mater. 2005, 17, 6479.

[18] H. Wei, S. M. Kauzlarich, Chem. Mater. 2006, 18, 1023.

[19] E. J. Henderson, C. M. Hessel, J. G. C. Veinot, J. Am. Chem. Soc. 2008, 130, 3624

[20] Z. C. Holma, U. R. Kortshagen, Langmuir 2009, 25, 11883.

[21] N. H. Chou, K. D. Oyler, N. E. Motl, R. E. Schaak, Chem. Mater. 2009, 21, 4105.

[22] D. D. Vaughn, J. F. Bondi, R. E. Sckaak, Chem. Mater. 2010, 22, 6103.

[23] X. M. Lu, B. A. Korgel, K. P. Johnston, Nanotechnology 2005, 16, S389.

[24] H. Gerung, S. D. Bunge, T. J. Boyle, C. J. Brinker, S. M. Han, Chem. Commun. 2005, 1914

[25] N. Zaitseua, Z. R. Dai, C. D. Grant, J. Harper, C. Saw, Chem. Mater. 2007, 19, 5174.
[26] A. M. Chockla, J. T. Harns, B. A. Korgel, Chem. Mater. 2011, 23, 1964.

[27] V. Belot, R. Corriu, D. Leclercq, P. M. Mutin, A. Vioux, Chem. Mater. 1991, 3, 127.

[28] H. A. Ketelson, M. A. Brook, R. Pelton, Y. M. Heng, Chem. Mater. 1996, 8, 2195.

[29] R. J. P. Corriu, D. Leclercq, Angew. Chem. Int. Ed. Engl. 1996, 35, 1420.

[30] L. Li, J. Shi, J. Yan, Chem. Commun. 2004, 1990.

[31] M. Cardona, Phys. Status Solidi 1983, 118, 463.

[32] E. B. Gorokhov, V. A. Volodin, D. V. Marin, D. A. Orekhov, A. G. Cherkov, A. K. Gutakovskii, V. A. Shvets, A. G. Borisov, M. D. Efremov, Semiconductors 2005, 39, 1168.

[33] J. Tauc, R. Grigorovici, A. Vancu, Phys. Status Solidi 1966, 15, 627.

[34] J. J. Singh, J. Non-Cryst. Solids 2002, 299-302, 444.

[35] M. Fujii, S. Hayashi, K. Yamamoto, Appl. Phys. Lett. 1990, 57, 2692.

[36] T. Hom, W. Kiszenick, B. Post, J. Appl. Cryst. 1975, 8, 457.

[37] Y. M. Niquet, G. Allan, C. Delerue, M. Lannoo, Appl. Phys. Lett. 2000, 77, 1182.

[38] S. H. Tang, E.Y. Chang, M. Hudait, J. S. Maa, C. W. Liu, G. L. Luo, H. D. Trinh, Y. H. Su, Appl. Phys. Lett. 2011, 98, 161905.

[39] Ö. Dag, E. J. Herderson, W. Wang, J. E. Lofgreen, S. Petrov, P. M. Brodersen, G. A. Ozin, J. Am. Chem. Soc. 2011, 133, 17454.

Received: November 24, 2011

Published online: January 9, 2012 